\title{
The Association of $\mathrm{Ki}-67$ and the Stage change between AJCC $7^{\text {th }}$ and $8^{\text {th }}$ Edition in Breast Cancer
}

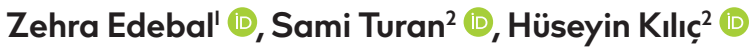 \\ 'Department of Pathology, Near East University School of Dentistry, Nicosia, Cyprus \\ 2Department of Medical Pathology, Konya Beyhekim State Hospital, Konya, Turkey
}

ORCID iDs of the authors: Z.E. 0000-000I-65I8-5972; S.T. 0000-0003-49I0-767X; H.K. 0000-000I-8692-4447.

Cite this article as: Edebal Z, Turan S, Kılıç H. The Association of Ki-67 and the Stage change between AJCC 7th and 8th Edition in Breast Cancer. Cyprus J Med Sci 2020; 5(4): 289-94.

\section{BACKGROUND/AIMS}

The new pathological prognostic staging in the 8th edition of the American Joint Committee on Cancer (AJCC) Cancer Staging Manual uses biomarkers, such as estrogen receptor (ER), progesterone receptor (PR), and human epidermal growth factor 2 (HER2) for breast cancer staging, but not $\mathrm{Ki}-67$. This study was designed to evaluate the relationship of $\mathrm{Ki}-67$ with pathological prognostic staging parameters and its possible correlation with this new staging system.

\section{MATERIAL and METHODS}

We performed a retrospective analysis on 59 invasive ductal breast carcinoma patients. We restaged all the patients using anatomic staging (AS) and pathological prognostic staging (PPS). The correlation of Ki-67 with ER, PR, HER2, histological grade, tumor size, and lymph node status were compared using the Chi-square test.

\section{RESULTS}

When patients classified according to AS were restaged using PPS, 21 (36\%) retained their original stage, while 34 ( $58 \%$ ) were downstaged and $4(6 \%)$ were upstaged. There was no correlation between the stage change and Ki-67, HER2, tumor grade, or size. Both, ER and PR positivity were markedly higher in the downstaged group ( $p=0.014$ and $p<0.001)$. Ki- 67 was not significantly different between AS patients; however, stage 3 PPS patients had a significantly more positive Ki-67 ratio than stage-I and stage-2 patients ( $p=0.007$ ). Moreover, Ki-67 had a significant negative correlation with ER and PR and positive correlation with the tumor grade, HER2, and lymph node involvement.

\section{CONCLUSION}

$\mathrm{Ki}-67$ is not useful for predicting the staging change from AS to PPS. However, it is strongly correlated with markers related to the biological features and prognosis in breast cancer. In order to increase its usefulness, more comprehensive studies are required.

Keywords: Breast cancer, Ki-67 antigen, cancer staging, biomarkers, prognosis

\section{INTRODUCTION}

Breast cancer is one of the most common malignancies across the world. About 2 million new cases are detected every year, and one of every 4 newly diagnosed cancer cases is that of breast cancer (I).

Until recently, the staging system developed by the American Joint Committee on Cancer (AJCC) that relies on the tumor size, lymph node involvement, and distant metastasis (TNM) was used for breast cancer management and prognosis estimation. With a deeper understanding of the biological factors related to breast cancer, the determination of various biomarkers has become a necessity (2). Thus, The AJCC Breast Cancer Expert Panel described a new "prognostic staging" that considers factors, such as estrogen receptor (ER), progesterone receptor (PR), and human epidermal growth factor receptor 2 (HER2) in addition to the TNM classification. They published this new staging system in the AJCC Cancer Staging Manual in 2016 and revised it in $2017(3,4)$. The pathological prognostic staging (PPS) is applicable for every patient who has undergone surgical excision for initial treatment without neoadjuvant therapy. This new staging is applied by using primary tumor size, lymph node involvement, distant metastasis, histological grade, ER, PR, 
and HER2. The AJCC Breast Cancer Expert Panel also recommended that a proliferation marker, such as Ki-67 and a genetic prognostic panel be performed at the time of initial diagnosis, if available (4). Ki-67 is an antigen expressed in the Gl, S, G2, and $M$ phases of the cell cycle, but not G0. The most common method for determining the $\mathrm{Ki}-67$ status is immunohistochemistry $(5,6)$. Although $\mathrm{Ki}-67$ is a recommended biomarker for assessing the proliferation status, it is not implemented in the PPS because it does not possess sufficient reliability owing to reproducibility issues and lack of agreement for cut-off points (4). Moreover, the results of the studies performed to establish a valid relationship between $\mathrm{Ki}-67$ and other PPS biomarkers have been inconsistent $(5,7-10)$.

Here, we aimed to investigate the relationship between $\mathrm{Ki}-67$ and other pathological prognostic factors in breast cancer as well as examine the effects of the $7^{\text {th }}$ and $8^{\text {th }}$ AJCC classifications on the staging change in the same patient. We also aimed to determine whether $\mathrm{Ki}-67$ or any other biomarkers used for classification affect the staging changes.

\section{MATERIALS and METHODS}

\section{Study Group and Pathological Evaluation}

Our study protocol was approved by the Health Sciences Ethical Committee of Near East University, with approval number YDU/2019/70-853. As our study was a retrospective trial and did not involve the use of personal data, the need for informed consent was waived off. We retrospectively collected the data of patients who were operated at the Konya Beyhekim State Hospital between January 2014 and May 2019 for invasive ductal carcinoma. We excluded patients who were in the carcinoma-in-situ stage or had distant metastasis, were missing pathologic prognostic staging biomarkers, had received neoadjuvant therapy, or had not undergone lymph node dissection. The histological grades, ER, PR, HER2, and Ki-67 statuses of the patients were reevaluated by using the existing slides in the same pathology laboratory by the three pathologists. Grading was performed following the Elston/Nottingham modification of the Bloom-Richardson system (Scarff- Bloom-Richardson Grading system, Nottingham Modification) by rating the following three morphological features: tubule formation, nuclear pleomorphism, and mitotic figure count of the tumor. Each parameter was assigned a score from I to 3, and the tumor was classified as grade I, 2, or 3, if the sum of these was 3-5, 6-7, and $8-9$, respectively. Staining over $1 \%$ was accepted as positive for

\section{Main Points:}

- Estrogen receptor (ER), Progesterone receptor (PR), and human epidermal growth factor receptor 2 (HER2) are included in the pathological prognostic for staging (PPS) breast cancer, but not $\mathrm{Ki}-67$.

- $\mathrm{Ki}-67$ positivity is significantly increased in PPS stage 3 patients.

- $\mathrm{Ki}-67$ is not useful for predicting the staging change from anatomic staging to PPS. However, $\mathrm{Ki}-67$ has a significant positive correlation with tumor grade, HER2, and lymph node involvement and it is also negatively correlated with $E R$ and $P R$.
ER and PR (II). For HER2, staining of 3+ was accepted as positive (I2). We accepted the cut-off value for Ki-67 positivity as $20 \%$, following the 2013 International St. Gallen Expert Consensus (13). Thereafter, the patients were restaged as per both, the $7^{\text {th }}$ edition (anatomic staging, AS) and $8^{\text {th }}$ edition (pathological prognostic staging, PPS) of the AJCC Cancer Staging Manual.

\section{Statistical Analysis}

All the statistical analyses were performed using the Statistical Package for Social Sciences software version I5 (SPSS Inc., Chicago, IL, USA). Patient age is reported as mean and standard deviation; tumor size and the absolute value of $\mathrm{Ki}-67$ are reported as median and interquartile range values. Categorical variables are reported as frequencies and percentages. Parametric factors were compared using the t-test, and non-parametric factors were compared using the Mann Whitney $U$ test or Kruskal-Wallis analysis. Categorical factors were compared using the Chi-square test and Fisher's exact test, where appropriate. If the $p$-value was $<0.05$, it was regarded significant.

\section{RESULTS}

The study population comprised of 97 patients. In thirty-eight of these patients, either one or more of the main markers used for breast cancer staging (ER, PR, HER2, Ki-67) was not analyzed and these patients were excluded from the study. We evaluated the data of the remaining 59 patients. One of the subjects was a man. The mean patient age was $61.4 \mathrm{y}$, and only 4 were aged $<40 \mathrm{y}$. Table I shows the demographic and clinicopathological features of the patients, and Figure I shows the frequency distribution of $\mathrm{Ki}-67$ absolute values.

We restaged the patients who were previously categorized as per the $7^{\text {th }}$ edition of the AJCC Cancer Staging Manual using the PPS. The consistency rate of the new staging was $36 \%$ because 21 of the 59 patients remained in the same stage, while 34 (58\%) were downstaged by at least one step, and 4 (6\%) were upstaged. Stage $3 \mathrm{C}$ and $2 \mathrm{~A}$ patients exhibited a staging change

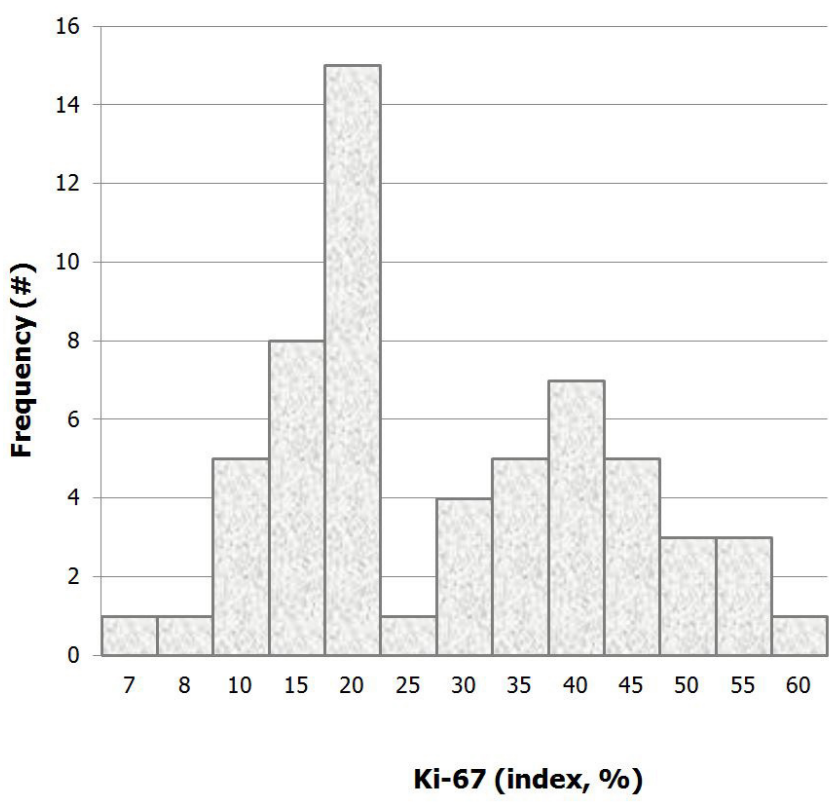

FIGURE I. Frequency distribution of the absolute Ki-67 values 


\begin{tabular}{|c|c|c|}
\hline & No. of Patients $(n=59)$ & $\%$ \\
\hline \multicolumn{3}{|l|}{ Localization } \\
\hline Left & 36 & 61 \\
\hline Right & 23 & 39 \\
\hline \multicolumn{3}{|l|}{ ER } \\
\hline Negative & 16 & 27 \\
\hline Positive & 43 & 73 \\
\hline \multicolumn{3}{|l|}{ PR } \\
\hline Negative & 24 & 41 \\
\hline Positive & 35 & 59 \\
\hline \multicolumn{3}{|l|}{ HR (ER or PR) } \\
\hline Negative & 13 & 22 \\
\hline Positive & 46 & 78 \\
\hline \multicolumn{3}{|l|}{ HER-2 } \\
\hline Negative & 45 & 76 \\
\hline Positive & 14 & 24 \\
\hline \multicolumn{3}{|c|}{ Histological Grade } \\
\hline Grade I & $\|$ & 19 \\
\hline Grade 2 & 35 & 59 \\
\hline Grade 3 & 13 & 22 \\
\hline \multicolumn{3}{|l|}{ Ki-67 } \\
\hline Negative & 30 & 51 \\
\hline Positive & 29 & 49 \\
\hline \multicolumn{3}{|c|}{ Lymph Node Metastasis } \\
\hline 0 & 18 & 31 \\
\hline I to 3 & 16 & 27 \\
\hline 4 to 9 & $\|$ & 19 \\
\hline 10 or more & 14 & 23 \\
\hline \multicolumn{3}{|l|}{ Tumor size } \\
\hline$\leq 2 \mathrm{~cm}$ & 19 & 32 \\
\hline \multirow[t]{2}{*}{$>2 \mathrm{~cm}$} & 40 & 68 \\
\hline & Mean & SD \\
\hline \multirow[t]{2}{*}{ Age (years) } & 61,4 & 13,9 \\
\hline & Median & Interquartile Range \\
\hline Ki-67 (\% Index) & 20 & 25 \\
\hline Tumor Size $(\mathrm{cm})$ & 2,5 & 1,9 \\
\hline Node Count & 3 & 9 \\
\hline \multicolumn{3}{|c|}{$\begin{array}{l}\text { ER: Estrogen receptor } \\
\text { PR: Progesterone receptor } \\
\text { HER2: Human epidermal growth factor receptor- } 2 \text {. }\end{array}$} \\
\hline
\end{tabular}

most frequently. Detailed staging distribution of the patients before and after restaging is presented in Table 2.

When the patient group whose staging remained unchanged after restaging was compared with the groups with a changed staging, a significant difference was found between stages I, 2, and 3 of Anatomical Staging ( $p=0.018$, Table 3). Stage 2 and 3 patients had markedly more staging changes as compared to
stage-I patients ( $p=0.014$ and $p=0.001$, respectively). When the upstaged, downstaged, and unchanged cases were compared, the groups showed no difference in the tumor grade, tumor size, HER2, or Ki-67. However, the downstaged group had significantly more ER and PR positivity $(p=0.014$ and $p<0.001$ ). Furthermore, all 4 patients who were upstaged were PR negative; only I had ER positivity.

Ki-67 was not different among stage-l, stage-2, and stage-3 patients in AS; however, there was a significant difference between the stages in PPS. The Ki-67 positivity of the patients in PPS with stage 3 breast cancer was significantly higher than in those with stage I or $2(p=0.007)$. When compared to the other clinicopathological features, $\mathrm{Ki}-67$ had a significant negative correlation with ER and PR ( $p=0.015$ and $p=0.026)$, and positive correlation with HER2 $(p<0.001)$, histological grade $(p<0.00 I)$, and lymph node involvement ( $p=0.047$, Table 4$)$.

\section{DISCUSSION}

$\mathrm{Ki}-67$ is an important biomarker for understanding the biology and behavior in breast cancer. It has an established prognostic property, and the determination of $\mathrm{Ki}-67$ status is encouraged by the AJCC Breast Cancer Expert Panel even though it is not included in the PPS $(4,14)$. However, studies that have investigated the relationship between $\mathrm{Ki}-67$ and breast cancer biomarkers, such as ER, PR, and HER2, have produced conflicting results.

In the present study, we found that Ki-67 was correlated with ER and PR negativity, HER2 positivity, increased histological grade, and lymph node involvement. Yip et al. reported similar correlations with ER, grade, and HER2, but not ER. Contrary to our results, their findings showed a relationship between $\mathrm{Ki}-67$ and tumor size (10). There was a significant correlation between $\mathrm{Ki}-67$ and tumor size, ER, PR, and grade, but not HER2 (Marwah et al.) (9). Another study reported that $\mathrm{Ki}-67$ was related to the tumor grade, but not the tumor size (15). Ahmed et al. (8) reported findings similar to our findings in that $\mathrm{Ki}-67$ was inversely correlated to ER and PR and had a positive correlation with grade and HER2, with no correlation to the tumor size. In contrast to some of these results and our findings, Kamranzadeh et al. (5) stated that Ki-67 was not correlated to ER, PR, tumor grade, or HER2. We believe that one possible explanation for these conflicting results might be the cutoff values chosen for Ki-67 in these studies. Yip et al. and Ahmed et al. chose $14 \%$, Shetty et al. and Kamranzadeh et al. used 10\%, and Marwah et al. determined 2 decision points as $5 \%$ and $20 \%$, for grouping the Ki-67 values. We used $20 \%$ as the cut-off point as per the recommendations of the International $\mathrm{Ki}-67$ in Breast Cancer Working Group and the 2013 St. Gallen consensus $(13,16)$. There is no universal agreement with respect to the cut-off point for $\mathrm{Ki}-67$. There are different $\mathrm{Ki}-67$ cut-off points in various studies, from 5\% to $34 \%$ (17). Moreover, Ki-67 has different cut-off values that have the same clinical significance in certain clinical conditions. Denkert et al. (18) reported that many different Ki-67 cut-off values have similar significance for evaluating disease-free survival, response to neoadjuvant therapy, and overall survival, and it is impossible to state which cut-off value is the most appropriate. We presume that it may be beneficial to specify different Ki67 cut-off values for different clinical purposes. Another reason for these conflicting results may be the differences in the study designs and variations in the patient attributes, such as mean age or race. Prospective and retrospective study designs might 
TABLE 2. Distribution of upstaging and downstaging in patients with anatomic staging and pathological prognostic staging

\begin{tabular}{|c|c|c|c|c|c|c|c|c|c|c|c|c|c|c|c|c|}
\hline \multirow[b]{3}{*}{ Anatomic Staging } & \multicolumn{16}{|c|}{ Pathological Prognostic Staging } \\
\hline & \multicolumn{2}{|c|}{ IA } & \multicolumn{2}{|c|}{ IB } & \multicolumn{2}{|c|}{$2 \mathrm{~A}$} & \multicolumn{2}{|c|}{$2 \mathrm{~B}$} & \multicolumn{2}{|c|}{$3 \mathrm{~A}$} & \multicolumn{2}{|c|}{ 3B } & \multicolumn{2}{|c|}{$3 C$} & \multicolumn{2}{|c|}{ Total } \\
\hline & No. & $\%$ & No. & $\%$ & No. & $\%$ & No. & $\%$ & No. & $\%$ & No. & $\%$ & No. & $\%$ & No. & $\%$ \\
\hline IB & & & 1 & 1.7 & I & 1.7 & & & & & & & & & 2 & 3.4 \\
\hline $2 \mathrm{~A}$ & 2 & 3.4 & 7 & 11.9 & 6 & 10.2 & 1 & 1.7 & & & & & & & 16 & 27.1 \\
\hline $3 \mathrm{~A}$ & & & & & 5 & 8.4 & 2 & 3.4 & 5 & 8.4 & । & 1.7 & & & 13 & 22.0 \\
\hline 3B & & & & & & & & & I & 1.7 & --- & 0 & & & I & 1.7 \\
\hline $3 C$ & & & & & & & & & & & 13 & 22.0 & 1 & 1.7 & 14 & 23.7 \\
\hline TOTAL & 7 & 11.8 & 10 & 17 & 15 & 25.4 & 6 & 10.2 & 6 & 10.2 & 14 & 23.7 & I & 1.7 & 59 & 100 \\
\hline
\end{tabular}

$\begin{aligned} & \text { TABLE 3. Rates of stage changes in patients classified as per ana- } \\
& \text { tomic staging }\end{aligned}$
\begin{tabular}{lcccc|}
$\begin{array}{l}\text { Anatomic } \\
\text { Stage }\end{array}$ & Unchanged & $\begin{array}{c}\text { Down } \\
\text { Staged }\end{array}$ & Up Staged & P* \\
\hline Stage I & $6(75 \%)$ & $0(0 \%)$ & $2(25 \%)$ & 0.003 \\
Stage II & $9(39 \%)$ & $13(57 \%)$ & $1(4 \%)$ & \\
Stage III & $6(21 \%)$ & $21(75 \%)$ & $1(4 \%)$ & \\
Total & $21(36 \%)$ & $24(58 \%)$ & $4(7 \%)$ & \\
\hline *Chi-square test & & & & \\
\hline
\end{tabular}

provide different outcomes; further, the inclusion of Caucasian, Asian, and/or Indian subjects in these studies may have caused the diverse results.

$\mathrm{Ki}-67$ is also an established prognostic marker that is important for distinguishing among certain types of breast cancer. This marker helps in determining the proliferative capacity of the tumor, an important characteristic of tumor biology. Therefore, its relation with the other biomarkers related to the biological status of the tumor has been a matter of concern. We found significant relationships between $\mathrm{Ki}-67$ status and ER negativity, PR negativity, HER2 positivity, increased histological grade, and lymph node involvement. First, mitotic index is one of the three parameters that are used for assigning the histological grade and is directly proportional to Ki-67. Thus, tumors with a higher mitotic index are expected to have a higher $\mathrm{Ki}-67$ value that manifests with a higher grade. In fact, several studies have reported a significant correlation between $\mathrm{Ki}-67$ and grade (8-I0, $15,19)$, while one research has shown a contradictory result (5). We found a strong positive correlation between Ki-67 and HER2 such that all the HER2 positive cases except one were also positive for Ki-67. Aziz et al., Yip et al., and Ahmed et al. state that HER2 positive tumors have a significantly higher Ki-67 value. In contrast, Kamranzadeh et al. and Marwah et al. reported that HER2 and Ki-67 were not significantly correlated. These studies used a cut-off value for $\mathrm{Ki}-67$, while the previous 3 studies compared the median $\mathrm{Ki}-67$ values of the groups. In addition, we found a significant correlation between $\mathrm{Ki}-67$ and hormone receptor negativity. Following the implementation of PPS in daily clinical practice, patients with a positive hormone receptor have usually been assigned to lower stages. With the help of targeted therapy, these markers exert a favorable effect on prognosis.
The negative prognostic features of $\mathrm{Ki}-67$ have been reported in various studies $(15,19,20)$. Although we were unable to evaluate its effect in this regard, we presume that the inverse relationship of Ki-67 with favorable biomarkers present in our study supports its undesirable effect on patient prognosis.

In our study, we inspected the agreement between AS and PPS as well as the factors that may affect the staging consistency. We found that $36 \%$ of our patients remained in the same stage, $58 \%$ were downstaged by at least one step, and $6 \%$ were upstaged. The consistency rate of restaging from the $7^{\text {th }}$ edition to the $8^{\text {th }}$ edition of the AJCC varied from $40.63 \%$ to $54.5 \%$ in other studies (7, 21-24). Most of these studies have reported upstaging rates of $5.3 \%-9.9 \%$ and downstaging rates were varying between $35.6 \%$ and $48.2 \%$ (21-24). But one study reported a much higher upstaging rate of $39.76 \%$ and a lower downstaging rate of $19.61 \%$ (7). Our upstaging rate was slightly higher than those reported by these studies. This could be because our patient population had a markedly higher mean age than the subjects in these studies. Moreover, most of our patients were in stage 3 , making up $47 \%$ of all our patients; $78 \%$ of these patients were assigned to a lower stage after restaging. In the other studies, $13 \%-21 \%$ of the patients were in stage 3 . We think that the high mean age of our study population and the high rate of advanced-stage patients compared to the aforementioned studies can be suggested as factors that influence our consistency and restaging rates.

AS expected, ER and PR were significantly different in the unchanged, upstaged, and downstaged groups. However, Ki-67, HER2 status, tumor grade, and tumor size were similar in these groups. Thus, we concluded that Ki-67 did not have any power in predicting the staging change. We performed the same analysis using $10 \%, 14 \%, 25 \%$, and $34 \%$ values for $\mathrm{Ki}-67$, as stated in other studies; however, we did not find a significant correlation between $\mathrm{Ki}-67$ and the staging change (data not shown). This finding is in contradiction with the findings reported by Ding et al (7). In the mentioned study, Ki-67, tumor size, and lymph node involvement were independent individual factors for predicting staging change. To our knowledge, no other single-center study has investigated the predictive ability of $\mathrm{Ki}-67$ on staging change from AS to PPS. We recommend that more comprehensive studies on larger patient populations be performed to evalvate this ability of Ki-67. 


\begin{tabular}{|c|c|c|c|}
\hline & \multicolumn{2}{|c|}{ Ki-67 } & \multirow[b]{2}{*}{$\mathbf{p}^{\dagger}$} \\
\hline & $\leq 20$ & $>20$ & \\
\hline \multicolumn{4}{|l|}{ Age } \\
\hline$\leq 50$ & $6(38 \%)$ & $10(62 \%)$ & \multirow[t]{2}{*}{0.211} \\
\hline$>50$ & $24(56 \%)$ & $19(44 \%)$ & \\
\hline \multicolumn{4}{|l|}{ Localization } \\
\hline Left & $19(53 \%)$ & $17(47 \%)$ & \multirow[t]{2}{*}{0.792} \\
\hline Right & II (48\%) & $12(52 \%)$ & \\
\hline \multicolumn{4}{|l|}{ ER } \\
\hline Negative & $4(25 \%)$ & $12(75 \%)$ & \multirow[t]{2}{*}{$0.015^{*}$} \\
\hline Positive & $26(61 \%)$ & $17(39 \%)$ & \\
\hline \multicolumn{4}{|l|}{ PR } \\
\hline Negative & $8(33 \%)$ & $16(67 \%)$ & \multirow[t]{2}{*}{$0.026^{*}$} \\
\hline Positive & $22(63 \%)$ & $13(37 \%)$ & \\
\hline \multicolumn{4}{|l|}{ HER-2 } \\
\hline Negative & $29(64 \%)$ & $16(36 \%)$ & \multirow[t]{2}{*}{$<0.00 l^{*}$} \\
\hline Positive & $\mathrm{I}(7 \%)$ & $13(93 \%)$ & \\
\hline \multicolumn{4}{|l|}{ Grade } \\
\hline I & $5(46 \%)$ & $6(54 \%)$ & \multirow[t]{3}{*}{$<0.00 l^{*}$} \\
\hline 2 & $25(71 \%)$ & $10(29 \%)$ & \\
\hline 3 & $0(0 \%)$ & $13(100 \%)$ & \\
\hline \multicolumn{4}{|c|}{ Anatomic Stage } \\
\hline Stage I & $5(63 \%)$ & $3(37 \%)$ & \multirow[t]{3}{*}{0.086} \\
\hline Stage II & $15(65 \%)$ & $8(35 \%)$ & \\
\hline Stage III & $10(36 \%)$ & $18(64 \%)$ & \\
\hline \multicolumn{4}{|c|}{ Pathologic Prognostic Stage } \\
\hline Stage I & $12(71 \%)$ & $5(29 \%)$ & \multirow[t]{3}{*}{$0.007^{*}$} \\
\hline Stage II & $13(62 \%)$ & $8(38 \%)$ & \\
\hline Stage III & $5(24 \%)$ & $16(76 \%)$ & \\
\hline \multicolumn{4}{|l|}{ Tumor Stage } \\
\hline $\mathrm{TI}$ & $13(68 \%)$ & $6(32 \%)$ & \multirow[t]{3}{*}{0.168} \\
\hline $\mathrm{T} 2$ & $14(44 \%)$ & $18(56 \%)$ & \\
\hline T3 & $3(38 \%)$ & $5(62 \%)$ & \\
\hline \multicolumn{4}{|l|}{ Node Status } \\
\hline NO & II (73\%) & $4(27 \%)$ & \multirow[t]{4}{*}{$0.047^{*}$} \\
\hline $\mathrm{NI}$ & $9(53 \%)$ & $8(47 \%)$ & \\
\hline N2 & $7(54 \%)$ & $6(46 \%)$ & \\
\hline N3 & $3(21 \%)$ & II (79\%) & \\
\hline $\begin{array}{l}\text { *Statistically } \\
{ }^{+} \text {Chi-square }\end{array}$ & & & \\
\hline
\end{tabular}

Lymph node involvement is one of the most important prognostic factors in breast cancer. However, some patients with similar tumor size and lymph node involvement have completely different prognosis (25). Thus, presenting the relationship of biomarkers with the nodal status might be beneficial for prognostic grouping. We demonstrated a significant positive correlation between $\mathrm{Ki}-67$ and lymph node involvement. Most of our NO cases were negative for $\mathrm{Ki}-67$, and the rate of the patients in $\mathrm{NI}$ and $\mathrm{N} 2$ stages did not have a meaningful difference from $\mathrm{NO}$ cases in terms of Ki-67 positivity. However, most of the N3 patients were Ki-67 positive. This implies that tumors with a high proliferation rate are prone to lymphatic spread. This correlation between $\mathrm{Ki}-67$ and lymph node involvement confirms that $\mathrm{Ki}-67$ is an important prognostic biomarker. But, our finding contradicts certain recent reports $(5,9,19)$. Nonetheless, a review of early breast cancer has shown that studies with a higher number of patients tend to demonstrate a significant positive correlation between Ki-67 and positive lymph node count (17). These contradictory results indicate the need for more comprehensive and larger population-based studies for investigating the correlation of $\mathrm{Ki}-67$ with lymph node status in breast cancer.

In the present study, the $\mathrm{Ki}-67$ positivity did not vary significantly in stages I, 2, and 3 in AS; however, we found that stage 3 patients in PPS had markedly higher Ki-67 positivity than stage-I and stage-2 patients. $\mathrm{Ki}-67$ is a proliferation marker with widespread availability and ease of application; however, the lack of reproducibility and universal cut-off value do not allow its implementation in PPS. Nevertheless, $\mathrm{Ki}-67$ is recommended by the expert panel to be determined at the time of initial diagnosis as a proliferation marker (4). Denkert et al. (14) recommended that the best strategy to demonstrate tumor biology in the adjuvant settings is to use $\mathrm{Ki}-67$ as a continuous marker, rather than as a cut-off. Based on this information, we also compared the median values of $\mathrm{Ki}-67$ among stage-l, stage-2, and stage- 3 patients in AS. We did not find any difference using a cut-off value as stated before, and stage 3 patients had a significantly higher $\mathrm{Ki}$ 67 median value than stage-I and stage-2 patients in AS (Kruskal-Wallis test, $p=0.043$, data not shown). We believe that this finding supports the suggestion of Denkert et al. and that using $\mathrm{Ki}-67$ as a continuous marker may be a better approach. Denkert et al. (14) also suggested that especially intermediate Ki-67 levels that have low analytic validity have limited applicability in clinical practice and that we should not determine whether Ki67 is positive based on marginal differences. Almost $50 \%$ of our patients had $\mathrm{Ki}-67$ values of $15 \%-25 \%$; this finding is important because it shows that this situation affects a large population of breast cancer patients.

This was a single-center study; this is a major strength of our study. Every sample was prepared in the same laboratory and evaluated by all three pathologists at the same time, minimizing the aforementioned variability of $\mathrm{Ki}-67$ staining. In addition, to our knowledge, our study is one of the few single-center studies to evaluate the new PPS and its effects as well as the relationship of $\mathrm{Ki}-67$ with the biomarkers used for PPS in a Turkic population.

This study has certain limitations; first, we studied a relatively small population because the study was performed at a single institution, and because our hospital does not specialize in breast care. In addition, the study population was relatively old and was diagnosed late for breast cancer; therefore, our sample was not representative of all breast cancer patients and was formed majorly of subjects who were in advanced stages. This may have influenced our consistency and restaging rates. Finally, we did not study the effects of the biomarkers on the prognosis, given our study design. Further, prognosis evaluation would have significantly contributed to our findings. 
In conclusion, $\mathrm{Ki}-67$ is not useful for predicting the staging change between AS and PPS. However, it is significantly correlated with most biomarkers used for PPS, emphasizing its importance in understanding the biological behavior of the tumor. Moreover, its correlation with stage and lymph node involvement strengthens its prognostic features. However, there remains a need for more comprehensive studies based on larger populations to increase the usefulness of $\mathrm{Ki}-67$ in understanding breast cancer biology.

Ethics Committee Approval: Ethics committee approval was received for this study from the Health Sciences Ethical Committee of the Near East University (YDU/2019/70-853).

\section{Informed Consent: N/A}

Peer-review: Externally peer-reviewed.

Author contributions: Concept - Z.E.; Design - Z.E.; Supervision - Z.E., H.K., S.T.; Resource - Z.E., H.K., S.T.; Materials - Z.E., H.K., S.T; Data Collection and/or Processing - Z.E., H.K., S.T.; Analysis and/or Interpretation - Z.E.; Literature Search - Z.E.; Writing - Z.E.; Critical Reviews - Z.E., H.K., S.T.

Acknowledgements: The authors want to acknowledge the efforts of Mehmet Kul for retrieving archived patient data and organizing pathological reports.

Conflict of Interest: Authors have no conflicts of interest to declare.

Financial Disclosure: The authors declared that this study has received no financial support.

\section{REFERENCES}

I. Bray F, Ferlay J, Soerjomataram I, Siegel RL, Torre LA, Jemal A. Global cancer statistics 2018: GLOBOCAN estimates of incidence and mortality worldwide for 36 cancers in 185 countries. CA Cancer J Clin 2018; 68(6): 394-424. [Crossref]

2. Giuliano AE, Connolly JL, Edge SB, Mittendorf EA, Rugo HS, Solin $\mathrm{LJ}$, et al. Breast Cancer-Major changes in the American Joint Committee on Cancer eighth edition cancer staging manual. CA Cancer J Clin 2017; 67(4): 290-303. [Crossref]

3. Hortobagyi GN, Connolly JL, D'Orsi CJ, Edge SB, Mittendorf EA, Rugo HS, et al. Breast. In: Amin MB, Edge S, Greene F, Byrd DR, Brookland RK, Washington MK, et al., editors. AJCC Cancer Staging Manual. 8 ed: Springer International Publishing; 2016. p. 589-636. [Crossref]

4. Updated Breast Chapter for 8th edition. American Joint Committee on Cancer; 10.1I.2017 [updated 13.03.2018; cited 01.08.2019]; Available from: http://cancerstaging.org/references-tools/deskreferences/ pages/breast-cancer-staging.aspx.

5. Kamranzadeh H, Ardekani RM, Kasaeian A, Sadighi S, Maghsudi S, Jahanzad I, et al. Association between Ki-67 expression and clinicopathological features in prognosis of breast cancer: A retrospective cohort study. J Res Med Sci 2019; 24: 30-7. [Crossref]

6. Clarke RB, Howell A, Potten CS, Anderson E. Dissociation between steroid receptor expression and cell proliferation in the human breast. Cancer Res 1997; 57(22): 4987-91.

7. Ding J, Wu W, Fang J, Chu Y, Zheng S, Jiang L. Changes of breast cancer staging when AJCC prognostic staging manual is used: a retrospective analysis of a Chinese cohort. Int J Biol Markers 2018; 33(2): 168-73. [Crossref]

8. Ahmed ST, Ahmed AM, Musa DH, Sulayvani FK, Al-Khyatt M, Pity IS. Proliferative Index (Ki67) for Prediction in Breast Duct Carcinomas. Asian Pac J Cancer Prev 2018; 19(4): 955-9.

9. Marwah N, Batra A, Marwah S, Gupta V, Shakya S, Sen R. Correlation of proliferative index with various clinicopathologic prognostic parameters in primary breast carcinoma: A study from North India. J Cancer Res Ther 2018; 14(3): 537-42. [Crossref]

10. Yip CH, Bhoo-Path N, Daniel JM, Foo YC, Mohamed AK, Abdullah MM, et al. Roles of Ki67 in Breast Cancer - Important for Management? Asian Pac J Cancer Prev 2016; 17: 1077-82. [Crossref]

II. Hammond MEH, Hayes DF, Wolff AC, Mangu PB, Temin S. American Society of Clinical Oncology/College of American Pathologists Guideline Recommendations for Immunohistochemical Testing of Estrogen and Progesterone Receptors in Breast Cancer. JCO Oncology Practice 2010; 6(4): 195-7. [Crossref]

12. Yaziii H, Goldstein LC, Barry TS, Werling R, Hwang H, Ellis GK, et al. HER-2 Testing in Breast Cancer Using Parallel Tissue-Based Methods. JAMA 2004; 29I(16): 1972-7. [Crossref]

13. Goldhirsch A, Winer EP, Coates AS, Gelber RD, Piccart-Gebhart $M$, Thürlimann B, et al. Personalizing the treatment of women with early breast cancer: highlights of the St Gallen International Expert Consensus on the Primary Therapy of Early Breast Cancer 2013. Annals of Oncology 2013; 24(9): 2206-23. [Crossref]

14. Denkert C, Budczies J, von Minckwitz G, Wienert S, Loibl S, Klauschen F. Strategies for developing $\mathrm{Ki} 67$ as a useful biomarker in breast cancer. Breast 2015; 24 Suppl 2: S67-72. [Crossref]

15. Shetty J, Rao C. Expression of E cadherin and Ki 67: Emerging Prognostic Markers in Triple-Negative Breast Cancer. Indian J Surg Oncol 2019; I0(2): 377-81. [Crossref]

16. Dowsett M, Nielsen TO, A'Hern R, Bartlett J, Coombes RC, Cuzick J, et al. Assessment of Ki67 in Breast Cancer: Recommendations from the International Ki67 in Breast Cancer Working Group. J Natl Cancer Inst 20Il; 103(22): 1656-64. [Crossref]

17. Urruticoechea A, Smith IE, Dowsett M. Proliferation marker Ki-67 in early breast cancer. J Clin Oncol 2005; 23(28): 7212-20. [Crossref]

18. Denkert C, Loibl S, Müller BM, Eidtmann H, Schmitt WD, Eiermann W, et al. Ki67 levels as predictive and prognostic parameters in pretherapeutic breast cancer core biopsies: a translational investigation in the neoadjuvant GeparTrio trial. Annals of Oncology 2013; 24(II): 2786-93. [Crossref]

19. Aziz S, Wik E, Knutsvik G, Klingen TA, Chen Y, Davidsen B, et al. Evaluation of Tumor Cell Proliferation by Ki-67 Expression and Mitotic Count in Lymph Node Metastases from Breast Cancer. PloS one 2016; II(3): e0150979. [Crossref]

20. de Azambuja E, Cardoso F, de Castro G Jr., Colozza M, Mano MS, Durbecq $\vee$, et al. $\mathrm{Ki}-67$ as prognostic marker in early breast cancer: a meta-analysis of published studies involving 12,155 patients. $\mathrm{Br} \mathrm{J}$ Cancer 2007; 96(10): 1504-13. [Crossref]

21. Wong RX, Wong FY, Lim J, Lian WX, Yap YS. Validation of the AJCC 8th prognostic system for breast cancer in an Asian healthcare setting. Breast 2018; 40: 38-44. [Crossref]

22. Shao N, Xie C, Shi Y, Ye R, Long J, Shi H, et al. Comparison of the 7th and 8th edition of American Joint Committee on Cancer (AJCC) staging systems for breast cancer patients: a Surveillance, Epidemiology and End Results (SEER) Analysis. Cancer Manag Res 2019; II: |433-42. [Crossref]

23. Jang N, Choi JE, Kang SH, Bae YK. Validation of the pathological prognostic staging system proposed in the revised eighth edition of the AJCC staging manual in different molecular subtypes of breast cancer. Virchows Arch 2019; 474(2): 193-200. [Crossref]

24. Ibis K, Ozkurt S, Kucucuk S, Yavuz E, Saip P. Comparison of Pathological Prognostic Stage and Anatomic Stage Groups According to the Updated Version of the American Joint Committee on Cancer (AJCC) Breast Cancer Staging 8th Edition. Med Sci Monit 2018; 24: 3637-43. [Crossref]

25. Yang ZJ, Yu Y, Hou XW, Chi JR, Ge J, Wang X, et al. The prognostic value of node status in different breast cancer subtypes. Oncotarget 2017; 8(3): 4563-7I. [Crossref] 